\title{
Multi-source TDOA estimation in reverberant audio using angular spectra and clustering ${ }^{1}$
}

\author{
Charles Blandin, Alexey Ozerov and Emmanuel Vincent \\ INRIA, Centre de Rennes - Bretagne Atlantique \\ Campus de Beaulieu, 35042 Rennes Cedex, France \\ \{charles.blandin, alexey.ozerov, emmanuel.vincent\}@inria.fr
}

\begin{abstract}
We consider the problem of estimating the time differences of arrival (TDOAs) of multiple sources from a two-channel reverberant audio signal. While several clustering-based or angular spectrum-based methods have been proposed in the literature, only relatively small-scale experimental evaluations restricted to either category of methods have been carried out so far. We design and conduct the first large-scale experimental evaluation of these methods and investigate a two-step procedure combining angular spectra and clustering. In addition, we introduce and evaluate five new TDOA estimation methods inspired from signal-to-noise-ratio (SNR) weighting and probabilistic multi-source modeling techniques that have been successful for anechoic TDOA estimation and audio source separation. The results show that clustering-based methods do not improve upon angular spectrum-based methods. For $5 \mathrm{~cm}$ microphone spacing, the best TDOA estimation performance is achieved by one of the proposed SNR-based angular spectrum methods. For larger spacing, a variant of the generalized cross-correlation with phase transform (GCC-PHAT) method performs best.
\end{abstract}

Keywords: Multiple source localization, TDOA estimation, angular spectrum, clustering

\section{Introduction}

Recorded audio signals often result from the mixture of several sound sources. The problem of source localization consists of estimating the spatial

\footnotetext{
${ }^{1}$ This work was supported in part by the ECHANGE project, funded by ANR, and by the Quaero Programme, funded by OSEO.
} 
positions of the sources and has many applications such as video-conferencing, surveillance, or source separation $[1,2]$. When the signal is recorded by an array of sensors, this problem is often addressed by estimating the Time Difference Of Arrival (TDOA) of each source for each pair of sensors [3]. Localization performance then mostly depends on the accuracy of the estimated TDOAs [1]. In the following, we focus on TDOA estimation of two or more sources for a given pair of sensors in a reverberant environment. The estimation of the number of sources and the assessment of the resulting localization performance for different array geometries are left for later study.

Most TDOA estimation methods use the Short Time Fourier Transform (STFT) of the signal $[3,4,5]$. Let us denote by $\mathbf{x}(t, f)=\left[x_{1}(t, f), x_{2}(t, f)\right]^{T}$ and $s_{n}(t, f), n=1, \ldots, N$, the STFTs of the observed signals and the $n$-th source signal, where $t=1, \ldots, T$ and $f=1, \ldots, F$ are, respectively, time frame and frequency bin indices. With these notations, the source mixing process can be modeled as [6]

$$
\mathbf{x}(t, f)=\sum_{n=1}^{N} \mathbf{d}\left(f, \tau_{n}\right) s_{n}(t, f)+\mathbf{b}(t, f),
$$

where

$$
\mathbf{d}\left(f, \tau_{n}\right)=\left[1, e^{-2 i \pi f \tau_{n}}\right]^{T}
$$

is the so-called steering vector associated with the $n$-th source of TDOA $\tau_{n}$ (in seconds), and $\mathbf{b}(t, f)$ models the reverberant part of the signal and additive noise, if any.

We distinguish three general approaches for TDOA estimation. The simplest one consists of computing the TDOA of the mixture signal locally in each time-frequency bin and localizing the peaks of the resulting histogram $[7,8,9,10]$. This approach is restricted to closely spaced or binaural microphones, since in the case of widely spaced microphones local TDOA computation becomes ambiguous due to spatial aliasing [11]. The second approach consists of iteratively estimating the time-frequency bins associated to each source and the corresponding TDOAs by means of some clustering algorithm $[11,6,12]$. This approach can be used for any microphone spacing but is sensitive to the initialization of the parameters (i.e., clusters and TDOAs). The third approach $[3,13,5,14,15]$ consists of building for each time-frequency bin a function of TDOA that is likely to exhibit a large value for true TDOAs, and pooling it across the time-frequency plane so as to obtain a so-called angular spectrum. The source TDOAs are then estimated 
as the highest peaks of this angular spectrum. This approach works for any microphone spacing and does not need any initial guess of the TDOAs. However, the possible presence of secondary peaks can alter the estimation of the TDOAs. In this paper, we focus on the two latter approaches, namely clustering and angular spectrum, which are always applicable.

Two main gaps are found in the literature. First, only relatively smallscale experimental evaluations restricted to either approach have been carried out so far $[16,17,18]$ and little is known about the variation of performance with respect to the microphone spacing, the reverberation time, the number of sources, the chosen angular spectrum pooling function or the chosen clustering initialization method. Second, existing methods typically rely on the assumption that the sources are disjoint in the time-frequency plane and affect the same weight to all time-frequency bins, regardless of the fact that the associated spatial information is less accurate in the presence of overlapping sources or reverberation.

This article aims to fill in these gaps. We design and conduct a largescale evaluation of angular spectrum-based and clustering-based methods on 1482 different configurations and investigate the use of the former for the initialization of the latter. In addition, we introduce and evaluate five new TDOA estimation methods inspired from signal-to-noise ratio (SNR) weighting or probabilistic modeling techniques that have been successful for anechoic TDOA estimation [19, 20, 21], histogram-based reverberant TDOA estimation [10] or audio source separation [22, 23], but have not yet been explored for angular spectrum-based or clustering-based reverberant TDOA estimation. The proposed methods account for the presence of diffuse noise or interfering sources in each time-frequency bin and rely prioritarily on the time-frequency bins resulting from the direct sound of a single source. The code of all methods and the experimental data are available at http://bass-db.gforge.inria.fr/bss_locate/.

The rest of the paper is organized as follows. In section 2 , a short review of existing angular spectrum-based methods is given and the proposed SNR-based methods are introduced. Existing and proposed clustering-based methods are presented in section 3. The experimental evaluation is detailed in section 4 , and conclusions are drawn in section 5 .

\section{Angular spectrum-based methods}

The principle of angular spectrum-based methods is to construct a function $\phi(\tau)$ of TDOA $\tau$ whose peaks indicate the TDOAs of the sources. This is commonly achieved as follows. A local angular spectrum (or coherence 
measure) $\phi(t, f, \tau)$ is computed in each time-frequency bin $(t, f)$ for all discrete values of $\tau$ lying on a uniform grid in the range of possible TDOAs. This function is chosen so that it is likely to exhibit large values for the TDOAs of the sources which are active in this time-frequency bin. In order to robustify the estimation process and to overcome the spatial aliasing ambiguity occuring at high frequencies, the function $\phi(t, f, \tau)$ is summed over all frequencies. Then, it is reduced to a single dimension to obtain the angular spectrum from which the TDOAs are estimated. This is typically done by summing it over all time frames [3]:

$$
\phi^{\mathrm{sum}}(\tau)=\sum_{t=1}^{T} \sum_{f=1}^{F} \phi(t, f, \tau) .
$$

A limitation of this approach is that it makes it difficult to localize a source that is active only within few time frames due to the integration of irrelevant information when the source is inactive. This can be addressed by taking the maximum over all time frames instead [14]:

$$
\phi^{\max }(\tau)=\max _{t} \sum_{f=1}^{F} \phi(t, f, \tau) .
$$

Existing methods differ by the definition of the local angular spectrum function $\phi(t, f, \tau)$ and the choice of the pooling function, i.e., "sum" (3) or "max" (4). Alternative pooling functions consisting of taking the $p$-th largest value over all time frames [24] or the mean of the $p$ largest values decreased the average performance compared to the "max" in our experiments and are not considered hereafter.

\subsection{Popular existing methods}

Existing methods typically extract the spatial information in time-frequency bin $(t, f)$ from the empirical covariance matrix $\widehat{\mathbf{R}}_{\mathbf{x x}}(t, f)$ of the input signal, which can be computed in the neighborhood of each time-frequency bin $(t, f)$ as $[25]$

$$
\widehat{\mathbf{R}}_{\mathbf{x} \mathbf{x}}(t, f)=\frac{\sum_{t^{\prime}, f^{\prime}} w\left(t^{\prime}-t, f^{\prime}-f\right) \mathbf{x}\left(t^{\prime}, f^{\prime}\right) \mathbf{x}\left(t^{\prime}, f^{\prime}\right)^{H}}{\sum_{t^{\prime}, f^{\prime}} w\left(t^{\prime}-t, f^{\prime}-f\right)},
$$

where $w$ is a time-frequency windowing function of length $L_{f} \times L_{t}$ defining the size and the shape of the neighborhood, and $(\cdot)^{H}$ denotes the Hermitian 
transposition operator.

The generalized cross-correlation with phase transform (GCC-PHAT) method [3] is certainly the most popular angular spectrum-based method. Based on the assumption that the direct sound of one source predominates in each time-frequency bin, the TDOA of this source $\tau$ is estimated from the phase difference between the two channels represented by the argument of $\widehat{\mathbf{R}}_{\mathbf{x x}}(t, f)_{1,2}{ }^{2}$. Indeed, this phase difference is expected to be close to $2 \pi f \tau$ modulo $2 \pi$. The local angular spectrum is then defined as

$$
\phi^{\mathrm{GCC}}(t, f, \tau)=\Re\left(\frac{\widehat{\mathbf{R}}_{\mathbf{x x}}(t, f)_{1,2}}{\left|\widehat{\mathbf{R}}_{\mathbf{x x}}(t, f)_{1,2}\right|} e^{-2 i \pi f \tau}\right),
$$

where $\Re(z)$ denotes the real part of a complex number $z$. In [24], it is proposed to use a non-linear function $\rho$ of $\phi^{G C C}(t, f, \tau)$ defined by $\rho(u)=$ $1-\tanh (\alpha \sqrt{1-u})$ in order to emphasize the large values of $\phi^{G C C}(t, f, \tau)$.

In multiple signal classification (MUSIC) [5], under the same assumption that one source is predominant in each time-frequency bin $(t, f)$, the local angular spectrum is computed as a measure of fit between the steering vector $\mathbf{d}(f, \tau)$ and the first principal component $\mathbf{v}(t, f)$ of $\widehat{\mathbf{R}}_{\mathbf{x x}}(t, f)$ :

$$
\phi^{\mathrm{MUSIC}}(t, f, \tau)=\left(1-\frac{1}{2}\left|\mathbf{d}(f, \tau)^{H} \mathbf{v}(t, f)\right|^{2}\right)^{-1},
$$

where $\mathbf{d}(f, \tau)$ is defined by $(2)$.

Nesta et al. [13] propose a method relaxing the assumption of one predominant source in each time-frequency bin. The time-frequency plane is split into time-frequency blocks, and it is assumed that there are at most two predominant sources in each block. Then, Independent Component Analysis (ICA) is applied in each time-frequency block $(t, f)$ to obtain two amplitudenormalized mixing coefficients $r_{1}(t, f)$ and $r_{2}(t, f)$ (see [13] for details) that are likely to be close to $e^{-2 i \pi f \tau_{1}}$ and $e^{-2 i \pi f \tau_{2}}$ up to a permutation, where $\tau_{1}$ and $\tau_{2}$ are the TDOAs of the two predominant sources in the considered block. The local angular spectrum of this method called cumulative state

\footnotetext{
${ }^{2}$ Here $\widehat{\mathbf{R}}_{\mathbf{x x}}(t, f)_{i, j}$ denotes the $(i, j)$-th element of matrix $\widehat{\mathbf{R}}_{\mathbf{x x}}(t, f)$.
} 
coherence transform (cSCT) is given by

$$
\phi^{\mathrm{cSCT}}(t, f, \tau)=\sum_{j=1}^{2} \rho\left(\frac{1}{2}\left|e^{-2 i \pi f \tau}-r_{j}(t, f)\right|\right),
$$

where $\rho(u)=1-\tanh (\alpha u)$.

\subsection{Proposed methods}

All existing methods, except the cSCT, use the assumption that in each time-frequency bin, only one source is predominant. For cSCT, this assumption is replaced by that of at most two predominant sources. Both assumptions do not hold exactly for most audio data [25, 26]. Thus, some of the estimated local angular spectra $\phi(t, f, \tau)$ do not represent any "true" TDOA possibly leading to poor estimation of the TDOAs. A bounded timefrequency weighting function based on interchannel correlation has been proposed in [27] to give more weight to the time-frequency bins involving a single predominant source. However, this function leads to overestimate the weight at low frequencies where interchannel correlation is large regardless of the number of active sources. Inspired by [19], where it was done for anechoic mixtures, we propose to use the SNR as an unbounded measure to determine whether the information contained in a time-frequency bin results from a single source. We propose three methods to estimate the SNR below.

\subsubsection{SNR estimation by beamforming}

In each time-frequency bin, we define the SNR in the direction corresponding to the TDOA $\tau$ by the ratio between the sound power in this direction and the residual power. We estimate the power in the direction corresponding to the TDOA $\tau$ by the Minimum Variance Distortionless Response (MVDR) beamformer [28]:

$$
P(t, f, \tau)=\left(\mathbf{d}(f, \tau)^{H} \widehat{\mathbf{R}}_{\mathbf{x x}}(t, f)^{-1} \mathbf{d}(f, \tau)\right)^{-1},
$$

where $\widehat{\mathbf{R}}_{\mathbf{x x}}(t, f)$ and $\mathbf{d}(f, \tau)$ are computed by (5) and (2), respectively. Then, we compute the residual power by subtracting the estimated power in the direction from an estimate of the total power: $\frac{1}{2} \operatorname{tr}\left(\widehat{\mathbf{R}}_{\mathbf{x x}}(t, f)\right)-P(t, f, \tau)$. Finally, we define the SNR in this direction as

$$
\phi^{\operatorname{MVDR}}(t, f, \tau)=\frac{\left(\mathbf{d}(f, \tau)^{H} \widehat{\mathbf{R}}_{\mathbf{x x}}(t, f)^{-1} \mathbf{d}(f, \tau)\right)^{-1}}{\frac{1}{2} \operatorname{tr}\left(\widehat{\mathbf{R}}_{\mathbf{x x}}(t, f)\right)-\left(\mathbf{d}(f, \tau)^{H} \widehat{\mathbf{R}}_{\mathbf{x x}}(t, f)^{-1} \mathbf{d}(f, \tau)\right)^{-1}} .
$$



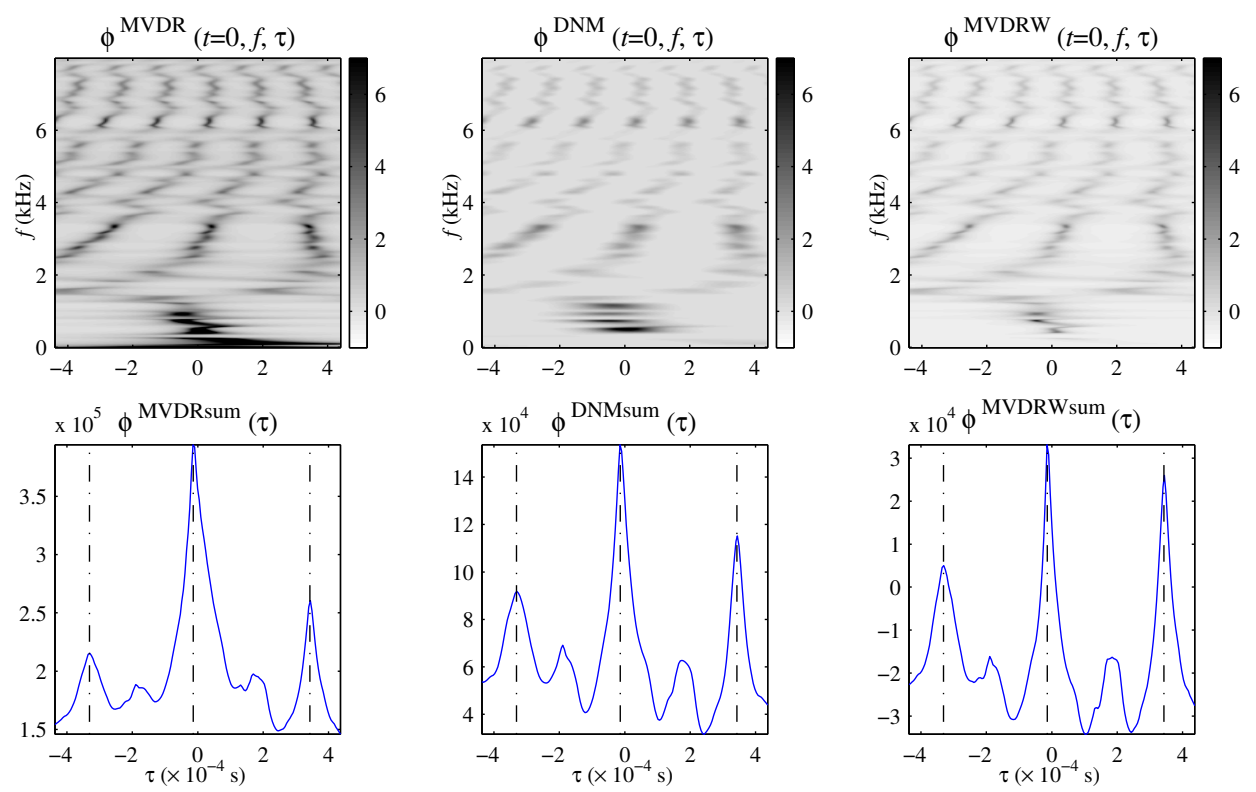

Figure 1: Local angular spectra $\phi(t, f, \tau)$ plotted at time $t=0$ (top) and global angular spectra $\phi^{\text {sum }}(\tau)$ pooled over all time frames (bottom) for $\phi^{\text {MVDR }}$ (left), $\phi^{\text {DNM }}$ (center) and $\phi^{\text {MVDRW }}$ (right), computed from the mixture of three female speech sources placed at 50 $\mathrm{cm}$ from the center of the microphone pair, with $d=15 \mathrm{~cm}$ and a reverberation time of $500 \mathrm{~ms}$. Dash-dotted lines indicate the true TDOAs.

Our preliminary experiments showed that this function overestimates the SNR at low frequencies, where phase differences are small, regardless of the number of active sources. As a consequence, the values of $\phi^{\mathrm{MVDR}}(t, f, \tau)$ at low frequencies mask the values at higher frequencies (see Fig. 1, left).

\subsubsection{SNR estimation under a diffuse noise model}

To address this problem, we propose to jointly estimate the sound power in direction $\tau$ and the power of the residual signal by using a diffuse noise model. We assume that in each time-frequency bin only one source $s(t, f)$ of TDOA $\tau$ is predominant, i.e., the mixing model (1) becomes

$$
\mathbf{x}(t, f)=\mathbf{d}(f, \tau) s(t, f)+\mathbf{b}(t, f) .
$$

Moreover, we assume that $s(t, f)$ and $\mathbf{b}(t, f)$ follow independent zero-mean Gaussian distributions with, respectively, variance $v^{s}(t, f, \tau)$ and covariance $v^{b}(t, f, \tau) \boldsymbol{\Psi}(f)$. The variances $v^{s}(t, f, \tau), v^{b}(t, f, \tau)>0$ represent the source 
and noise power, and $\boldsymbol{\Psi}(f)$ is the covariance matrix of a gain-normalized diffuse noise $[6,18]$ :

$$
\Psi(f)=\left(\begin{array}{cc}
1 & \operatorname{sinc}\left(2 \pi f \frac{d}{c}\right) \\
\operatorname{sinc}\left(2 \pi f \frac{d}{c}\right) & 1
\end{array}\right),
$$

with $d$ being the distance between the two microphones (in meters), $c$ the speed of sound (in meters/sec.), and $\operatorname{sinc}(y)=\frac{\sin (y)}{y}$ the cardinal sine function. With these assumptions, it can be shown from (11) that the covariance matrix of the mixture in the time-frequency bin $(t, f)$ and for TDOA $\tau$ can be expressed as

$$
\mathbf{R}_{\mathbf{x x}}(t, f, \tau)=v^{s}(t, f, \tau) \mathbf{d}(f, \tau) \mathbf{d}^{H}(f, \tau)+v^{b}(t, f, \tau) \Psi(f),
$$

and the log-likelihood of $\mathbf{x}(t, f)$ can be written as:

$$
\begin{aligned}
\log p(\mathbf{x}(t, f)) & =\log N\left(\mathbf{x}(t, f) ; 0, \mathbf{R}_{\mathbf{x x}}(t, f, \tau)\right) \triangleq \\
& -\operatorname{tr}\left(\mathbf{R}_{\mathbf{x x}}^{-1}(t, f, \tau) \widehat{\mathbf{R}}_{\mathbf{x x}}(t, f)\right)-\log \operatorname{det}\left(\pi \mathbf{R}_{\mathbf{x x}}(t, f, \tau)\right) .
\end{aligned}
$$

Using a closed form solution from [18], we estimate $v^{s}(t, f, \tau)$ and $v^{b}(t, f, \tau)$ in the maximum likelihood sense as

$$
\left(\begin{array}{c}
v^{s}(t, f, \tau) \\
v^{b}(t, f, \tau)
\end{array}\right)=\left(\operatorname{diag}\left(\boldsymbol{\Lambda}_{1}\right), \operatorname{diag}\left(\boldsymbol{\Lambda}_{2}\right)\right)^{-1} \operatorname{diag}\left(\mathbf{A}^{-1} \widehat{\mathbf{R}}_{\mathbf{x x}}(t, f)\left(\mathbf{A}^{H}\right)^{-1}\right),
$$

where $(\mathbf{Y}, \mathbf{Z}$ ) denotes the concatenation of matrices (or vectors) $\mathbf{Y}$ and $\mathbf{Z}$, $\operatorname{diag}(\mathbf{Y})$ denotes the column vector of diagonal entries of matrix $\mathbf{Y}, \mathbf{A}$ is the matrix whose columns are the eigenvectors of $\mathbf{d}(f, \tau) \mathbf{d}^{H}(f, \tau) \mathbf{\Psi}^{-1}(f)$, and $\boldsymbol{\Lambda}_{1}, \boldsymbol{\Lambda}_{2}$ are equal respectively to $\mathbf{A}^{-1} \mathbf{d}(f, \tau) \mathbf{d}^{H}(f, \tau)\left(\mathbf{A}^{H}\right)^{-1}$ and $\mathbf{A}^{-1} \mathbf{\Psi}(f)\left(\mathbf{A}^{H}\right)^{-1}$. Non-negativity of $v^{s}(t, f, \tau)$ and $v^{b}(t, f, \tau)$ is imposed by setting $v^{s}(t, f, \tau)$ to zero and $v^{b}(t, f, \tau)$ to $\frac{1}{2} \operatorname{tr}\left(\Psi^{-1}(f) \widehat{\mathbf{R}}_{\mathbf{x x}}(t, f)\right)$ when $v^{s}(t, f, \tau)$ or $v^{b}(t, f, \tau)$ resulting from (15) is negative [18]. Finally, the SNR in the time-frequency bin $(t, f)$ and for TDOA $\tau$ is computed as

$$
\phi^{\mathrm{DNM}}(t, f, \tau)=\frac{v^{s}(t, f, \tau)}{v^{b}(t, f, \tau)} .
$$

This method effectively addresses the SNR overestimation problem at low frequencies but results in slightly wider peaks (see Fig. 1, center). 


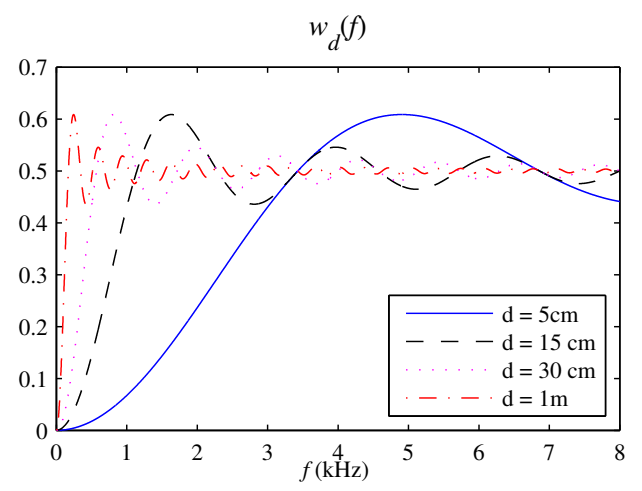

Figure 2: Weighting factor $w_{d}(f)$ for microphone spacings $d=5 \mathrm{~cm}, 15 \mathrm{~cm}, 30 \mathrm{~cm}$ and 1 m.

\subsubsection{SNR estimation by frequency weighted beamforming}

To take advantage of both the precision of $\phi^{\text {MVDR }}$ (see Fig. 1, left) and the global shape of $\phi^{\mathrm{DNM}}$ (see Fig. 1, center), we combine these two methods by expressing a relationship between them, assuming that the input signal consists of a single source of TDOA $\tau=0$ and diffuse noise. In other words, we replace in (10) the empirical covariance matrix $\widehat{\mathbf{R}}_{\mathbf{x x}}(t, f)$ by the covariance matrix $\mathbf{R}_{\mathbf{x x}}(t, f, \tau)$ in (13), and we get

$$
\phi^{\mathrm{MVDR}}(t, f, \tau)=\frac{1+2 v^{s}(t, f, \tau) / v^{b}(t, f, \tau)+\operatorname{sinc}\left(2 \pi f \frac{d}{c}\right)}{1-\operatorname{sinc}\left(2 \pi f \frac{d}{c}\right)} .
$$

By inverting (17), we estimate the SNR as:

$$
\phi^{\mathrm{MVDRW}}(t, f, \tau)=\frac{v^{s}(t, f, \tau)}{v^{b}(t, f, \tau)}=w_{d}(f) \phi^{\mathrm{MVDR}}(t, f, \tau)+w_{d}(f)-1,
$$

where $w_{d}(f)=\frac{1}{2}\left(1-\operatorname{sinc}\left(2 \pi f \frac{d}{c}\right)\right)$ is a weighting factor depending on the frequency and the microphone spacing $d$. This factor reduces the impact of low frequencies, as it can be seen from Figure 2 and from Figure 1 (top right) below $1 \mathrm{KHz}$. It can be also noted from Figure 1 that $\phi^{\mathrm{MVDRW}}$ has sharper peaks than both $\phi^{\mathrm{MVDR}}$ and $\phi^{\mathrm{DNM}}$.

\section{Clustering-based methods}

In angular spectrum-based methods a measure of source activity (e.g., the SNR) can be exploited, but the estimation of this measure does not rely 
on any estimates of the TDOAs. In contrast, the principle of clusteringbased methods consists of estimating this measure given some estimates of the TDOAs, then reestimating the TDOAs relying on this measure, and so on. This results in iterating between the following two steps:

- re-estimate the contribution of each source to each time-frequency bin (clusters), given current estimates of the TDOAs,

- re-estimate the TDOAs, given current estimates of the clusters.

\subsection{Popular existing methods}

Sawada et al. [11] propose to perform the clustering in a hard manner, by associating each time-frequency bin to the closest source $n_{t f}$ in the sense of the Euclidean distance between its steering vector and the phase and amplitude-normalized observation. Each TDOA $\tau_{n}$ is then reestimated from the time-frequency bins of the corresponding cluster only.

It is also possible to perform the clustering in a soft manner in a probabilistic setting. Araki et al. [29] assume that the phase difference between the two channels follows a wrapped Gaussian distribution for each source and each time-frequency bin. This results in a Gaussian mixture model (GMM), whose parameters are estimated via the Expectation-Maximization (EM) algorithm. The E-step achieves soft clustering by estimating the probability of $n_{t f}$. This method also includes a sparsity-inducing prior on the GMM mixture weights enabling the estimation of the number of sources.

Pointing that the Euclidean distance and the Gaussian distribution over the phase differences do not accurately represent the effect of multiple sources and reverberation, Izumi et al. [6] adopt the following model instead:

$$
\mathbf{x}(t, f)=s_{n_{t f}}(t, f) \mathbf{d}\left(f, \tau_{n_{t f}}\right)+\mathbf{b}(t, f) .
$$

The source coefficients $s_{n_{t f}}(t, f)$ are considered as deterministic parameters and the noise term $\mathbf{b}(t, f)$ is assumed to be diffuse and modeled as a zeromean Gaussian random vector of covariance matrix $v^{b} \boldsymbol{\Psi}(f)$, where $\boldsymbol{\Psi}(f)$ is given by (12) and $v^{b}$ is a constant parameter. Parameter estimation is achieved via the EM algorithm again.

\subsection{Proposed methods}

3.2.1. EM algorithm with one predominant source in each time-frequency bin

In line with [6], we assume that in each time-frequency bin $(t, f)$, there is only one predominant source $n_{t f}$ and a diffuse noise $\mathbf{b}(t, f)$. However, 
in contrast to [6], we assume that both $s_{n_{t f}}(t, f)$ and $\mathbf{b}(t, f)$ follow independent zero-mean Gaussian distributions with covariances $v_{n_{t f}}^{s}(t, f)$ and $v_{n_{t f}}^{b}(t, f) \boldsymbol{\Psi}(f)$, where $v_{n_{t f}}^{s}(t, f)$ and $v_{n_{t f}}^{b}(t, f)$ represent respectively the source and the noise variances, and $\boldsymbol{\Psi}(f)$ is defined by (12). Given the predominant source index $n_{t f}$, the mixture covariance matrix is $\mathbf{R}_{\mathbf{x x}, n_{t f}}(t, f)=$ $\boldsymbol{\Xi}_{\mathbf{x}}\left(f, \tau_{n_{t f}}, v_{n_{t f}}^{s}(t, f), v_{n_{t f}}^{b}(t, f)\right)$, where

$$
\boldsymbol{\Xi}_{\mathbf{x}}\left(f, \tau, v^{s}, v^{b}\right) \triangleq v^{s} \mathbf{d}(f, \tau) \mathbf{d}^{H}(f, \tau)+v^{b} \boldsymbol{\Psi}(f) .
$$

The set of parameters to be estimated is

$$
\theta=\left\{\left\{\tau_{n}\right\}_{n=1}^{N},\left\{v_{n}^{s}(t, f), v_{n}^{b}(t, f)\right\}_{n, t, f=1}^{N, T, F}\right\} .
$$

Under the above assumptions and assuming a uniform prior over the source indices, the observation $\mathbf{x}(t, f)$ follows the Gaussian mixture model (GMM) distribution

$$
p(\mathbf{x}(t, f) \mid \theta)=\sum_{n} \frac{1}{N} p\left(\mathbf{x}(t, f) \mid \tau_{n}, v_{n}^{s}(t, f), v_{n}^{b}(t, f)\right),
$$

where

$$
p\left(\mathbf{x}(t, f) \mid \tau, v^{s}, v^{b}\right)=N\left(\mathbf{x}(t, f) ; 0, \mathbf{\Xi}_{\mathbf{x}}\left(f, \tau, v^{s}, v^{b}\right)\right),
$$

and $N\left(\mathbf{x}(t, f) ; 0, \boldsymbol{\Xi}_{\mathbf{x}}\left(f, \tau, v^{s}, v^{b}\right)\right)$ is defined by (14).

We use an EM algorithm [30] to estimate the parameters $\theta$ in the maximum likelihood sense, considering the set of predominant source indices $\left\{n_{t f}\right\}_{t, f}$ as latent data. The updates of the resulting algorithm and some hints for its derivation are given in Appendix A.

\subsubsection{EM algorithm with multiple sources in each time-frequency bin}

As it was already mentioned in section 2.2 , the assumption of only one predominant source does not hold exactly for most audio data. Several works $[25,26,22,23]$ have shown that relaxing this assumption can be very beneficial for audio source separation. Thus, we here investigate whether such an approach could be beneficial for multi-source localization. The model presented below is mostly inspired by the models proposed in $[22,23]$. We consider that all sources can be present in each time-frequency bin and model the mixing process as

$$
\mathbf{x}(t, f)=\mathbf{D}(f, \boldsymbol{\tau}) \mathbf{s}(t, f)+\mathbf{b}(t, f),
$$


where $\boldsymbol{\tau}=\left[\tau_{1}, \ldots, \tau_{N}\right]$ is the vector of TDOAs, $\mathbf{D}(f, \boldsymbol{\tau})=\left[\mathbf{d}\left(f, \tau_{1}\right), \ldots, \mathbf{d}\left(f, \tau_{N}\right)\right]$, $\mathbf{s}(t, f)=\left[s_{1}(t, f), \ldots, s_{N}(t, f)\right]^{T}$ and $\mathbf{b}(t, f)=\left[b_{1}(t, f), b_{2}(t, f)\right]^{T}$.

We assume that $\mathbf{b}(t, f)$ and $\mathbf{s}(t, f)$ follow zero-mean Gaussian distributions with covariance matrices respectively equal to $v^{b}(t, f) \Psi(f)$ and $\mathbf{R}_{\mathbf{s s}}(t, f)$, where $\mathbf{R}_{\mathbf{s s}}(t, f)$ is the diagonal matrix with $n$-th diagonal entry being equal to $v_{n}^{s}(t, f)$. With these assumptions the observation $\mathbf{x}(t, f)$ follows a zero-mean Gaussian distribution with covariance matrix

$$
\mathbf{R}_{\mathbf{x x}}(t, f)=\mathbf{D}(f, \boldsymbol{\tau}) \mathbf{R}_{\mathbf{s s}}(t, f) \mathbf{D}^{H}(f, \boldsymbol{\tau})+v^{b}(t, f) \Psi(f) .
$$

The set of parameters to be estimated is

$$
\theta=\left\{\left\{\tau_{n}\right\}_{n=1}^{N},\left\{v_{n}^{s}(t, f)\right\}_{n, t, f=1}^{N, T, F},\left\{v^{b}(t, f)\right\}_{t, f}^{T, F}\right\}
$$

To estimate the parameters in the maximum likelihood sense we derive an EM algorithm, considering the sources $\{\mathbf{s}(t, f)\}_{t, f}$ as latent data. The algorithm is summarized in Appendix B. We consider four variants of the algorithm:

1. "EM-multi (TF noise)": the noise variances $v^{b}(t, f)$ are unconstrained,

2. "EM-multi (F noise)": in line with [22], the noise variances $v^{b}(t, f)$ are constrained to be constant over time, i.e., $v^{b}(t, f)=v^{b}(f)$,

3. "EM-multi (const noise)": in line with [6], the noise variances $v^{b}(t, f)$ are constrained to be constant over time and frequency, i.e., $v^{b}(t, f)=$ $v^{b}$,

4. "EM-multi (no noise)": in line with [11], the noise variances $v^{b}(t, f)$ are fixed to a small positive value $\varepsilon^{\text {fix }}$.

\section{Evaluation}

We performed a large-scale evaluation of the methods presented in this article. The data, the evaluation measures and the code of the tested methods are available on http://bass-db.gforge.inria.fr/bss_locate/.

\subsection{Data}

The experimental evaluation was carried out on a large number of mixtures of male speech, female speech and music sources taken from the database of the 2008 Signal Separation Evaluation Campaign (SiSEC) [31] "underdetermined speech and music mixtures" task. Mixing filters were simulated 
with the Roomsimove Toolbox ${ }^{3}$ for a rectangular room of dimensions 4.45 $\mathrm{m} \times 3.55 \mathrm{~m} \times 2.5 \mathrm{~m}$ and omnidirectional microphones. We considered all possible combinations of the following parameters:

- Number of sources $N$ : from 2 to 6 .

- Reverberation time $\mathrm{RT}_{60}{ }^{4}$ : $50 \mathrm{~ms}, 100 \mathrm{~ms}, 150 \mathrm{~ms}, 250 \mathrm{~ms}, 500 \mathrm{~ms}$, $750 \mathrm{~ms}$.

- Microphone spacing $d: 5 \mathrm{~cm}, 15 \mathrm{~cm}, 30 \mathrm{~cm}, 1 \mathrm{~m}$.

- Distance between the sources and the center of the microphone pair: $20 \mathrm{~cm}, 50 \mathrm{~cm}, 1 \mathrm{~m}, 2 \mathrm{~m}$.

- Angular position of the sources: between 3 and 5 randomly generated scenarios according to the number of sources, i.e., 5 scenarios for 2 sources, 4 for $3-4$ sources, and 3 for 5 - 6 sources. Moreover, the scenarios were generated with the following restrictions: the Directions Of Arrival (DOAs) cannot be smaller (greater) than 30 degrees (150 degrees) and the absolute difference between DOAs of any pair of sources cannot be smaller than 15 degrees for 2-5 sources and 10 degrees for 6 sources.

- Three source types (female speech, male speech and music).

We only kept situations for which the distance between the sources and the microphone pair is lower than $0.8 d$ to ensure that the far-field assumption holds, so that the relation between a TDOA $\tau$ and its corresponding DOA $\eta$ (in degrees) can be expressed by

$$
\tau=\frac{d}{c} \cos \left(2 \pi \frac{\eta}{360}\right)
$$

This resulted in a total of 4446 mixtures. All the signals were sampled at $16 \mathrm{kHz}$.

\footnotetext{
${ }^{3}$ E. Vincent, D. Campbell, Roomsimove, a Matlab toolbox for the computation of simulated room impulse reponses for moving sources, http://www.irisa.fr/metiss/members/ evincent/software.

${ }^{4} \mathrm{RT}_{60}$ is the time required for reflections of a direct sound to decay by $60 \mathrm{~dB}$ below the level of the direct sound.
} 


\subsection{Evaluation measures}

\subsubsection{Localization}

Evaluation in terms of localization consists of measuring the capacity of the methods to estimate the true TDOAs with some tolerance. Since we do not attempt to estimate the number of sources $N$, we apply the considered methods for all possible numbers of sources $J$ from 1 to 20 . For a given $J$, we select the $J$ highest peaks of the angular spectra and run the clustering algorithms with $J$ clusters. We then evaluate the list of $J$ estimated TDOAs compared to the list of $N$ true TDOAs in terms of recall, precision and Fmeasure. An estimated TDOA $\hat{\tau}$ is considered as correctly estimated if it is close enough to the true TDOA $\tau$, in the sense that the corresponding DOAs $\hat{\eta}$ and $\eta$, computed by inverting (27), differ by less than 5 degrees modulo 180 degrees. Denoting by $I_{J}$ the number of correctly estimated TDOAs, recall, precision and F-measure are defined by [32]

$$
\begin{gathered}
\operatorname{Recall}(J)=\frac{I_{J}}{N}, \\
\operatorname{Precision}(J)=\frac{I_{J}}{J}, \\
\text { F-measure }(J)=2 \times \frac{\operatorname{Recall}(J) \times \operatorname{Precision}(J)}{\operatorname{Recall}(J)+\operatorname{Precision}(J)} .
\end{gathered}
$$

Figure 3 shows the average recall, precision and F-measure of several angular spectrum-based methods over all mixtures with $N=3$ sources. The results are represented as a function of the assumed number of sources $J$. As expected, the recall increases and the precision decreases with $J$, while the F-measure is maximum for some $J=J_{\text {opt }}$. $J_{\text {opt }}$ equals to the number of sources $N$ for some method, but not for all. Thus, $J_{\text {opt }}$ depends on the method $\mathcal{A}$ and on the number of sources $N$. We observed a similar behavior for other methods and other numbers of sources. We also noticed that it depends on the microphone spacing $d$ but that it is insensitive to the other parameters. Thus, for each method $\mathcal{A}$, each number of sources $N$ and each microphone spacing $d$, we compute $J_{\text {opt }}(\mathcal{A}, N, d)$ that maximizes the average $\mathrm{F}$-measure over the corresponding mixtures. In the rest of this section, we assume that $J$ is fixed to $J_{\text {opt }}(\mathcal{A}, N, d)$ for all mixtures.

\subsubsection{Accuracy}

When the TDOAs are correctly estimated, one would like to know how accurate these estimates are. Let the TDOAs of all the mixtures considered be enumerated through as $\left\{\tau^{i}\right\}_{i=1}^{I}$. Let $\mathcal{I} \subset\{1, \ldots, I\}$ denote the subset of 

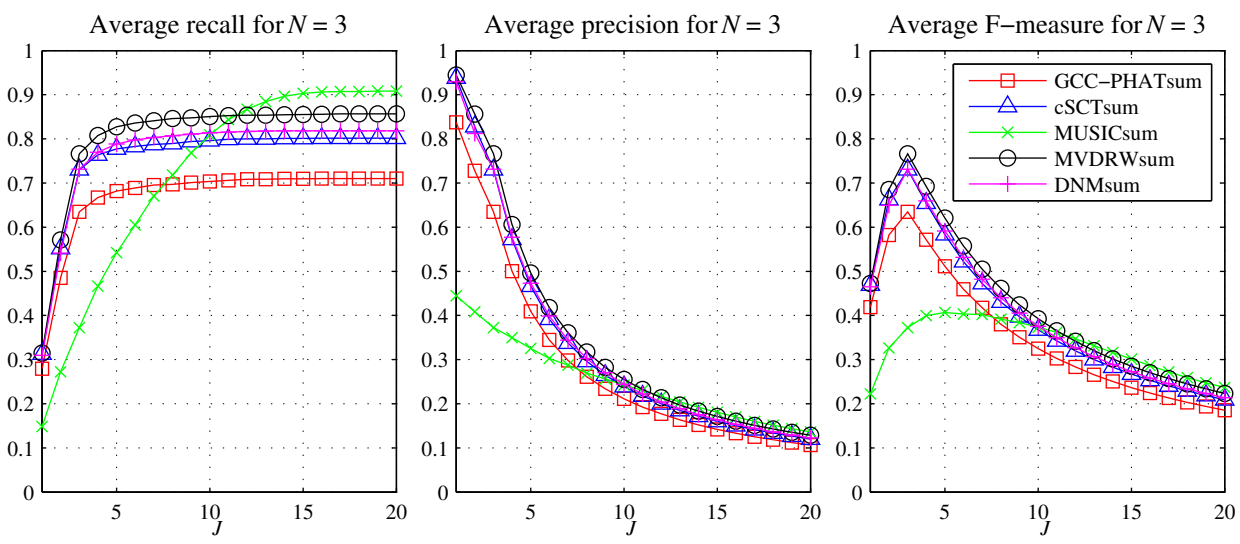

Figure 3: Average recall (left), precision (center) and F-measure (right) as functions of $J$ for several angular spectrum-based methods and for all mixtures with $N=3$ sources.

TDOAs that are correctly estimated by all the methods under comparison. We define the average accuracy as follows:

$$
\text { Accuracy }=\frac{1}{\sum_{i \in \mathcal{I}} N_{i}^{-1}} \sum_{i \in \mathcal{I}} N_{i}^{-1}\left|\left(\eta^{i}-\hat{\eta}^{i}\right) \bmod 180\right|,
$$

where $\eta^{i}$ and $\hat{\eta}^{i}$ are the DOAs computed from the true $\tau^{i}$ and estimated $\hat{\tau}^{i}$ TDOAs using (27), and $N_{i}$ denotes the number of sources in the corresponding mixture. Note that this measures the accuracy of only those TDOAs which are correctly estimated by all methods under consideration instead of each method individually.

\subsection{Parameters}

All the methods evaluated below had exactly the same front-end to compute the empirical covariance matrices $\widehat{\mathbf{R}}_{\mathbf{x x}}(t, f)(5)$, except the cSCT [13], for which we used an implementation provided by the author of [13]. The STFT was computed with half-overlapping sine windows of length 1024. The time-frequency windowing function $w$ in (5) was the outer product of two Hanning windows. Its size was set to $L_{f}=L_{t}=1$ for GCC-PHAT and GCC-NONLIN, $L_{f}=15$ and $L_{t}=3$ for the other angular spectrum-based methods and $L_{f}=L_{t}=3$ for all clustering-based methods, since this gave the best results in our preliminary experiments. 


\begin{tabular}{|c|c|c|c|c|c|c|c|c|}
\hline \multirow{2}{*}{$\begin{array}{l}\text { Local angular } \\
\text { spectrum function }\end{array}$} & \multicolumn{2}{|c|}{$\begin{array}{c}\text { Recall } \\
(0 \text { to } 1)\end{array}$} & \multicolumn{2}{|c|}{$\begin{array}{l}\text { Precision } \\
(0 \text { to } 1)\end{array}$} & \multicolumn{2}{|c|}{$\begin{array}{l}\text { F-measure } \\
(0 \text { to } 1)\end{array}$} & \multicolumn{2}{|c|}{$\begin{array}{l}\text { Accuracy } \\
\text { (degrees) }\end{array}$} \\
\hline & $\phi^{\text {sum }}$ & $\phi^{\max }$ & $\phi^{\text {sum }}$ & $\phi^{\max }$ & $\phi^{\text {sum }}$ & $\phi^{\max }$ & $\phi^{\text {sum }}$ & $\phi^{\max }$ \\
\hline GCC-PHAT [3] & 0.61 & 0.84 & 0.72 & 0.85 & 0.65 & 0.84 & - & 0.42 \\
\hline GCC-NONLIN [24] & 0.67 & 0.85 & 0.73 & 0.85 & 0.69 & 0.85 & - & 0.45 \\
\hline MUSIC [5] & 0.65 & 0.64 & 0.33 & 0.30 & 0.43 & 0.41 & - & - \\
\hline cSCT [13] & 0.67 & 0.82 & 0.74 & 0.83 & 0.70 & 0.82 & 0.83 & 0.53 \\
\hline MVDR & 0.73 & 0.80 & 0.75 & 0.80 & 0.74 & 0.80 & 0.56 & 0.48 \\
\hline DNM & 0.70 & 0.72 & 0.73 & 0.71 & 0.71 & 0.71 & 0.63 & 0.61 \\
\hline MVDRW & 0.73 & 0.80 & 0.75 & 0.80 & 0.74 & 0.80 & 0.56 & 0.48 \\
\hline
\end{tabular}

Table 1: Angular spectrum-based methods: average results for all 4446 mixtures. The average accuracy was computed over the 7755 TDOAs correctly estimated by the methods with average F-measure greater than 0.7 (from a total of 16614).

\subsection{Angular spectrum-based methods}

We consider four state-of-the-art local angular spectra: GCC-PHAT [3], a version of GCC-PHAT with a non-linear function [24] (denoted as GCC-NONLIN), MUSIC [5] and CSCT [13], and the three proposed SNRbased local angular spectra: MVDR, DNM and MVDRW (see Sec. 2.2). All seven local angular spectra were evaluated with both $\phi^{\text {sum }}(\cdot)(3)$ and $\phi^{\max }(\cdot)$ (4) pooling functions, except the $\operatorname{cSCT}[13]^{5}$. Note also that for all the methods, in line with [24], we do not select peaks that differ by less than 5 degrees from a higher peak.

The results in terms of average recall, precision, F-measure and accuracy are reported in Table 1 . Since the average accuracy is computed over the TDOAs that are correctly estimated by all the compared methods, as explained in section 4.2.2, we have selected for this comparison the methods that performed better than 0.7 in terms of average F-measure. This was done in order to avoid a comparison over a very small subset of TDOAs.

Analyzing the results in terms of F-measure, we see that for the "sum" pooling function MVDR and MVDRW outperform all other methods, which confirms our findings in [18]. Using the "max" pooling function instead of "sum" improves MVDR and MVDRW. However, it improves even more GCC-PHAT and GCC-NONLIN, which perform best in the end. The results in terms of average accuracy appear correlated with the average F-measure, and GCC-PHATmax is the most accurate on average.

\footnotetext{
${ }^{5}$ Since we have only an implementation of the cSCT [13] given us by the author of [13] without the corresponding sources, we were not able injecting the "max" pooling function into this implementation.
} 

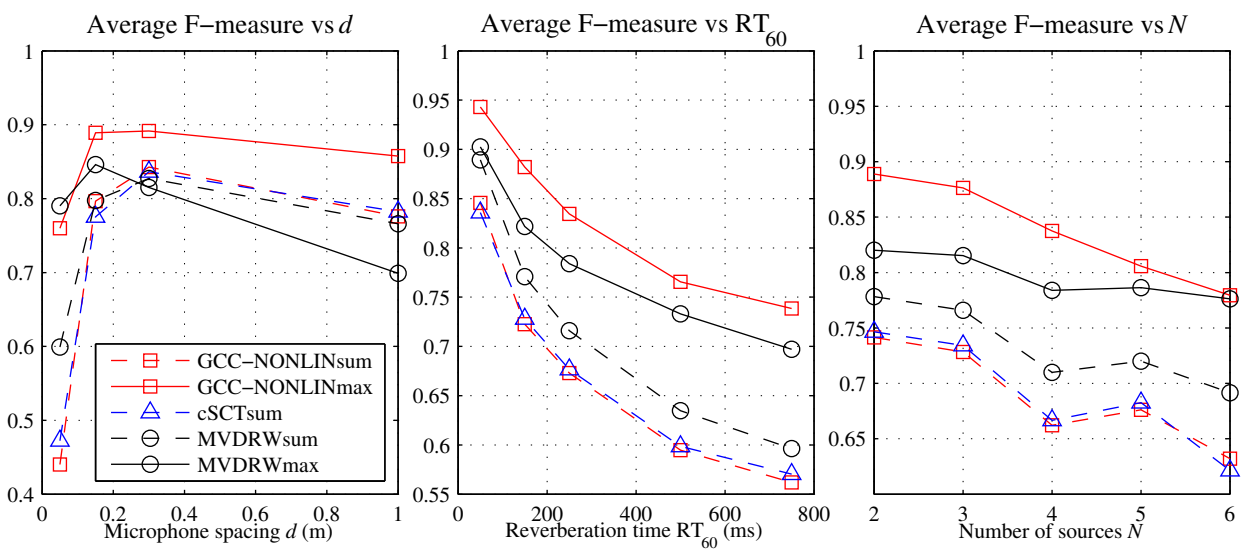

Figure 4: Angular spectrum-based methods: average F-measure as a function of the microphone spacing $d$ (left), the reverberation time $\mathrm{RT}_{60}$ (center), and the number of sources $N$ (right).

We then investigate the behaviour of the average F-measure as a function of the microphone spacing, the reverberation time and the number of sources. For this comparison we chose five methods among the best ones, namely GCC-NONLINsum, GCC-NONLINmax, cSCTsum, MVDRWsum and MVDRWmax. The results are shown in Figure 4. While the reverberation time and the number of sources have little influence on the ranking of different methods, the microphone spacing has. Note that in case of the "sum" pooling function the advantage of MVDRW against GCC-NONLIN was essentially due to its better performance for the $5 \mathrm{~cm}$ microphone spacing. Using the "max" pooling function improves GCC-NONLIN for all microphone spacings, but it improves MVDRW only for small spacings, while leading to a big performance degradation for large spacings. However, our proposed MVDRWmax method outperforms GCC-NONLINmax by $0.03 \mathrm{~F}$ measure for $5 \mathrm{~cm}$ microphone spacing. This setting is useful in practice, since it matches the size of handheld devices such as portable audio recorders and smartphones.

\subsection{Clustering-based methods}

The evaluation of clustering-based methods, as compared to that of angular spectrum-based methods, is much more computationally expensive within our evaluation framework. Indeed, as explained in Sec. 4.2.1, each clustering-based method must be run 20 times (for $J$ from 1 to 20) with 


\begin{tabular}{|c|c|c|c|c|c|c|c|c|}
\hline \multirow[t]{2}{*}{ Clustering algorithm } & \multicolumn{2}{|c|}{$\begin{array}{l}\text { Recall } \\
(0 \text { to } 1)\end{array}$} & \multicolumn{2}{|c|}{$\begin{array}{c}\text { Precision } \\
(0 \text { to } 1)\end{array}$} & \multicolumn{2}{|c|}{$\begin{array}{c}\text { F-measure } \\
(0 \text { to } 1)\end{array}$} & \multicolumn{2}{|c|}{$\begin{array}{l}\text { Accuracy } \\
\text { (degrees) }\end{array}$} \\
\hline & rand & init & rand & init & rand & init & rand & init \\
\hline None & 0.53 & 0.90 & 0.24 & 0.90 & 0.32 & 0.90 & - & 0.56 \\
\hline Sawada et al. [11] & 0.66 & 0.88 & 0.76 & 0.87 & 0.49 & 0.87 & - & 0.75 \\
\hline Izumi et al. [6] & 0.29 & 0.30 & 0.48 & 0.51 & 0.30 & 0.35 & - & - \\
\hline EM-predom & 0.66 & 0.84 & 0.81 & 0.85 & 0.52 & 0.85 & - & 0.66 \\
\hline EM-multi (TF noise) & 0.94 & 0.90 & 0.50 & 0.90 & 0.32 & 0.90 & - & 0.56 \\
\hline EM-multi (F noise) & 0.94 & 0.90 & 0.50 & 0.90 & 0.32 & 0.90 & - & 0.56 \\
\hline EM-multi (const noise) & 0.94 & 0.90 & 0.48 & 0.90 & 0.31 & 0.90 & - & 0.56 \\
\hline EM-multi (no noise) & 0.94 & 0.90 & 0.47 & 0.90 & 0.32 & 0.90 & - & 0.56 \\
\hline
\end{tabular}

Table 2: Clustering-based methods: average results for 1482 female speech mixtures. Notations: "rand" means initializing clustering by random TDOAs, "init" means initializing it by TDOAs estimated with GCC-NONLINmax, and "None" means evaluating the initialization without running any clustering algorithm. The average accuracy was computed over the 4384 TDOAs correctly estimated by the methods with average F-measure greater than 0.7 (from a total of 5538).

several EM iterations. Since we have not noticed any influence of signal type on the ranking of angular spectrum-based methods, we retain only the female speech mixtures for this evaluation.

We consider two state-of-the-art methods by Sawada et al. [11] and Izumi et al. [6], and the four of the proposed methods: the "EM-predom" method introduced in Sec. 3.2.1 and the variants of the "EM-multi" method introduced in Sec. 3.2.2. To evaluate the impact of initialization on the performance, each method was run twice: $(i)$ with randomly initialized TDOAs and $(i i)$ with initial TDOAs estimated by GCC-NONLINmax, i.e., the best angular spectrum-based method. For each mixture, the random initial TDOAs were drawn from the uniform distribution on the interval $[-d / c, d / c]$. For each method the source and the noise variances $v_{n}^{s}(t, f)$ and $v_{n}^{b}(t, f)$ were initialized by some constant values $v^{s, \text { init }}$ and $v^{b \text {,init }}$ adjusted during preliminary tests. Each method was run for a maximum of 100 iterations until the estimated TDOAs did not change from one iteration to the next.

The results in terms of average evaluation measures, including those of the initialization (noted by "None"), are summarized in Table 2. All methods fail when randomly initialized: the best results achieved by the proposed "EM-predom" method remain far below those of GCC-NONLINmax and all other methods except that Sawada et al. do not improve over mere random guess. The initialization provided by GCC-NONLINmax greatly improves the average F-measure for all methods. However, GCC-NONLINmax alone 

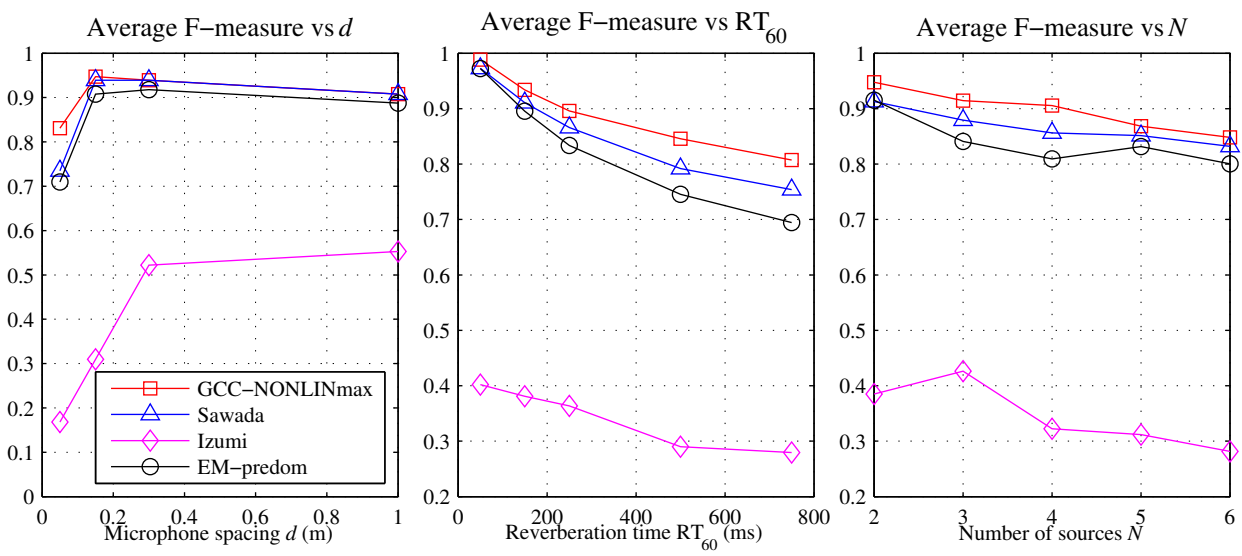

Figure 5: Clustering-based methods: average F-measure as a function of microphone spacing $d$ (left), reverberation time $\mathrm{RT}_{60}$ (center), and number of sources $N$ (right). All methods are initialized by the TDOAs estimated by GCC-NONLINmax.

is the most accurate in the end: the four "EM-multi" methods provide exactly the same results, while Sawada et al. and "EM-predom" result in a small performance degradation. This illustrates the fact that these methods remain stuck in the local optimum provided by GCC-NONLINmax, so that improved clustering techniques are needed to overcome this performance ceiling.

We choose the four distinct methods after proper initialization, namely GCC-NONLINmax, Sawada et al. [11], Izumi et al. [6] and "EM-predom", to investigate the behaviour of the average F-measure as a function of different parameters. The results are shown in Figure 5. We see that the clustering-based methods, as compared to GCC-NONLINmax used for the initialization, decrease the average F-measure for all considered conditions. Moreover, the drop in performance is mostly pronounced for small microphone spacings.

\section{Conclusion}

In this paper we introduced several multi-source TDOA estimation methods based on angular spectra and clustering. The common motivation behind all these methods is to go beyond the assumption of a single predominant source per time-frequency bin. This is achieved by either using the SNR as an unbounded measure of source activity or by exploiting directly 
the multi-source hypothesis within a probabilistic model. We evaluated the proposed and the five most popular state-of-the-art methods on 1482 different configurations. To our knowledge, this is by far the largest-scale evaluation of multi-source TDOA estimation methods to date.

Among all angular spectrum-based methods, the best performance was achieved by the proposed MVDRWmax method for $5 \mathrm{~cm}$ microphone spacing and by GCC-NONLINmax for larger spacings. The former setting is useful in practice, since it matches the size of handheld devices such as portable audio recorders and smartphones.

All clustering-based methods were evaluated with both random TDOA initialization and an initialization provided by the best angular spectrumbased method. First, we showed that it is very important to initialize the clustering algorithms with "good" TDOA estimates, since random initialization leads to really poor performance. Second, we observed that none of the clustering-based methods was able to improve the TDOA estimates compared to the best angular spectrum-based method.

Our findings show that SNR weighting and probabilistic modeling techniques that have been successful for anechoic TDOA estimation and audio source separation bring little or no improvement for reverberant TDOA estimation compared to GCC-PHAT, so that more specific approaches are needed to solve this problem in the future. The role of the "max" pooling function, its success and its potential limits should be better understood, so as to be able to propose more powerful non-linear pooling functions. The resulting localization performance should be assessed for different array geometries and the problem of estimating the number of sources remains open, with few methods proposed so far [29, 14, 24]. Finally, we still believe in the potential of clustering-based methods provided that the considered i.i.d. source variance models are replaced by more structured audio-specific models as in, e.g., [22]. An alternative way would be to try injecting the "max" pooling function within the probabilistic model behind clustering.

\section{Appendix A. Updates for the EM algorithm with one predomi- nant source in each time-frequency bin}

An EM algorithm [30] is an iterative algorithm consisting in updating the parameters $\theta^{(l)}$ at every iteration $l$ as follows:

$$
\theta^{(l)}=\arg \max _{\theta} Q\left(\theta, \theta^{(l-1)}\right),
$$


where $Q\left(\theta, \theta^{\prime}\right)$ is the so-called auxiliary function. Under the assumptions of Section 3.2.1 this function is equal (up to some additive constant) to

$$
Q\left(\theta, \theta^{\prime}\right) \stackrel{\mathrm{c}}{=} \sum_{n, t, f} \gamma_{n}(t, f) p\left(\mathbf{x}(t, f) \mid \tau_{n}, v_{n}^{s}(t, f), v_{n}^{b}(t, f)\right),
$$

with

$$
\begin{aligned}
\gamma_{n}(t, f) & \triangleq p\left(n_{t f}=n \mid \mathbf{x}(t, f), \theta^{\prime}\right) \\
& \propto p\left(\mathbf{x}(t, f) \mid \tau_{n}^{\prime}, v_{n}^{s \prime}(t, f), v_{n}^{b \prime}(t, f)\right),
\end{aligned}
$$

where, for any parameter $z$ from $\theta, z^{\prime}$ denotes the corresponding parameter from $\theta^{\prime}$ and $p\left(\mathbf{x}(t, f) \mid \tau, v^{s}, v^{b}\right)$ is defined by (23).

As before, the TDOAs are only estimated on the discrete uniform grid in the range of possible TDOAs. Let us denote this grid by $\Gamma$. One iteration of the EM parameter updates optimizing (A.1) for the auxiliary function (A.2) consists of the following steps:

1. Estimate $\hat{v}_{n}^{s}(t, f, \tau)$ and $\hat{v}_{n}^{b}(t, f, \tau)$ in the maximum likelihood sense for all time-frequency bins and all possible TDOAs $\tau \in \Gamma$ using (15) ${ }^{6}$.

2. Compute the posterior cluster probabilities $\gamma_{n}(t, f)$ as in (A.4) and normalize them so that $\sum_{n} \gamma_{n}(t, f)=1$.

3. Update the TDOAs $\tau_{n}$ as follows:

$$
\tau_{n}=\arg \max _{\tau \in \Gamma} \sum_{t, f} \gamma_{n}(t, f) \log p\left(\mathbf{x}(t, f) \mid \tau, \hat{v}_{n}^{s}(t, f, \tau), \hat{v}_{n}^{b}(t, f, \tau)\right),
$$

where $p\left(\mathbf{x}(t, f) \mid \tau, \hat{v}_{n}^{s}(t, f, \tau), \hat{v}_{n}^{b}(t, f, \tau)\right)$ is defined by (23).

4. Update the source and noise variances by setting $v_{n}^{s}(t, f)=\hat{v}_{n}^{s}\left(t, f, \tau_{n}\right)$ and $v_{n}^{b}(t, f)=\hat{v}_{n}^{b}\left(t, f, \tau_{n}\right)$.

5. Set $\theta^{\prime}=\theta$.

\section{Appendix B. Updates for the EM algorithm with multiple sources in each time-frequency bin}

Performing some derivations analogous to those from [22] and [23], it can be shown that the auxiliary function $Q\left(\theta, \theta^{\prime}\right)$ for the EM algorithm under

\footnotetext{
${ }^{6}$ Note that in practice this needs to be done only once for all iterations.
} 
assumptions of Section 3.2.2 is equal (up to some additive constant) to:

$$
\begin{aligned}
& Q\left(\theta, \theta^{\prime}\right) \stackrel{\mathrm{c}}{=} \\
- & \sum_{n, t, f}\left(\log v_{n}^{s}(t, f)+\frac{\widehat{\mathbf{R}}_{\mathbf{s s}}(t, f)_{n, n}}{v_{n}^{s}(t, f)}\right)-\sum_{t, f}\left(2 \log v^{b}(t, f)+\frac{\mathcal{M}\left(t, f, \boldsymbol{\tau}, \theta^{\prime}\right)}{v^{b}(t, f)}\right),
\end{aligned}
$$

where

$$
\begin{gathered}
\mathcal{M}\left(t, f, \boldsymbol{\tau}, \theta^{\prime}\right) \triangleq \operatorname{tr}\left[\Psi^{-1}(f) \widehat{\mathbf{R}}_{\mathbf{x x}}(t, f)-\Psi^{-1}(f) \mathbf{D}(f, \boldsymbol{\tau}) \widehat{\mathbf{R}}_{\mathbf{x \mathbf { s }}}^{H}(t, f)\right. \\
\left.-\boldsymbol{\Psi}^{-1}(f) \widehat{\mathbf{R}}_{\mathbf{x \mathbf { s }}}(t, f) \mathbf{D}^{H}(f, \boldsymbol{\tau})+\boldsymbol{\Psi}^{-1}(f) \mathbf{D}(f, \boldsymbol{\tau}) \widehat{\mathbf{R}}_{\mathbf{s}}(t, f) \mathbf{D}^{H}(f, \boldsymbol{\tau})\right] \\
\widehat{\mathbf{R}}_{\mathbf{x \mathbf { s }}}(t, f)=\widehat{\mathbf{R}}_{\mathbf{x x}}(t, f) \mathbf{G}_{\mathbf{s}}^{H}(t, f), \\
\widehat{\mathbf{R}}_{\mathbf{s} \mathbf{s}}(t, f)=\mathbf{G}_{\mathbf{s}}(t, f) \widehat{\mathbf{R}}_{\mathbf{x \mathbf { s }}}(t, f)+\left[\mathbf{I}_{N}-\mathbf{G}_{\mathbf{s}}(t, f) \mathbf{D}\left(f, \boldsymbol{\tau}^{\prime}\right)\right] \mathbf{R}_{\mathbf{s} \mathbf{s}}^{\prime}(t, f),
\end{gathered}
$$

with $\widehat{\mathbf{R}}_{\mathbf{x x}}(t, f)$ defined by (5), and

$$
\begin{gathered}
\mathbf{G}_{\mathbf{s}}(t, f)=\mathbf{R}_{\mathbf{s} \mathbf{s}}^{\prime}(t, f) \mathbf{D}^{H}\left(f, \boldsymbol{\tau}^{\prime}\right)\left(\mathbf{R}_{\mathbf{x x}}^{\prime}(t, f)\right)^{-1} \\
\mathbf{R}_{\mathbf{x} \mathbf{x}}^{\prime}(t, f)=\mathbf{D}\left(f, \boldsymbol{\tau}^{\prime}\right) \mathbf{R}_{\mathbf{s} \mathbf{s}}^{\prime}(t, f) \mathbf{D}^{H}\left(f, \boldsymbol{\tau}^{\prime}\right)+v^{b \prime}(t, f) \mathbf{\Psi}(f) .
\end{gathered}
$$

One iteration of the EM parameter updates optimizing (A.1) for the auxiliary function (B.1) consists of the following steps:

1. Compute $\widehat{\mathbf{R}}_{\mathbf{x x}}(t, f), \widehat{\mathbf{R}}_{\mathbf{x \mathbf { s }}}(t, f)$ and $\widehat{\mathbf{R}}_{\mathbf{s \mathbf { s }}}(t, f)$ using (5), (B.3) and (B.4).

2. Update the source variances as $v_{n}^{s}(t, f)=\widehat{\mathbf{R}}_{\mathbf{s s}}(t, f)_{n, n}$.

3. Update the TDOAs as $\tau_{n}=\tau_{n}^{*}$, where:

$$
\tau_{n}^{*}=\arg \min _{\tau_{n} \in \Gamma} \sum_{t, f} \frac{1}{v^{b}(t, f)} \mathcal{M}\left(t, f, \boldsymbol{\tau}, \theta^{\prime}\right),
$$

and $\mathcal{M}\left(t, f, \boldsymbol{\tau}, \theta^{\prime}\right)$ is defined by (B.2). More precisely, each TDOA $\tau_{n}$ $(n=1, \ldots, N)$ is updated in turn, while keeping the other TDOAs $\left\{\tau_{m}\right\}_{m \neq n}$ fixed ${ }^{7}$.

\footnotetext{
${ }^{7}$ Both the usage of the noise variances $v^{b}(t, f)$ in the updates of TDOAs (B.7) and the alternating nature of these updates do not guarantee the maximization of the auxiliary function, as in (A.1). However, they guarantee its non-decrease, i.e., $Q\left(\theta^{(l)}, \theta^{(l-1)}\right) \geq$ $Q\left(\theta^{(l-1)}, \theta^{(l-1)}\right)$. Thus, the resulting algorithm is rather a Generalized EM (GEM) algorithm [30]. Updating TDOAs jointly, instead of alternatively, is possible as well, but it is avoided here, since it would lead to a computational complexity growing exponentially with the number of sources.
} 
4. Update noise variances:

$$
v^{b}(t, f)=\frac{1}{2 \cdot \# \mathcal{J}(t, f)} \sum_{(\tilde{t}, \tilde{f}) \in \mathcal{J}(t, f)} \mathcal{M}\left(\tilde{t}, \tilde{f}, \boldsymbol{\tau}, \theta^{\prime}\right),
$$

with $\mathcal{J}(t, f) \subset\{1, \ldots, T\} \times\{1, \ldots, F\}$ denoting the subset of timefrequency bins where $v^{b}(t, f)$ is considered constant and $\# \mathcal{J}(t, f)$ denoting the number of elements in this subset. This formulation allows the implemention of the first three variants of the algorithm mentioned at the end of section 3.2.2. To implement the fourth variant one just needs to skip the noise variance update.

5. Set $\theta^{\prime}=\theta$.

\section{Acknowledgment}

The authors would like to thank Francesco Nesta for sharing his cSCT implementation, and Nobutaka Ito for discussion during the course of this work.

\section{References}

[1] M. Brandstein, D. Ward (Eds.), Microphone Arrays: Signal Processing Techniques and Applications, Springer, Berlin, 2001.

[2] S. Makino, H. Sawada, T.-W. Lee (Eds.), Blind Speech Separation, Springer, 2007.

[3] C. Knapp, G. Carter, The generalized cross-correlation method for estimation of time delay, IEEE Transactions on Acoustics, Speech and Signal Processing 24 (4) (1976) 320-327.

[4] O. Yılmaz, S. Rickard, Blind separation of speech mixtures via timefrequency masking, IEEE Transactions on Signal Processing 52 (7) (2004) 1830-1847.

[5] R. Schmidt, Multiple emitter location and signal parameter estimation, IEEE Transactions on Antennas and Propagation 34 (3) (1986) 276280.

[6] Y. Izumi, N. Ono, S. Sagayama, Sparseness-based 2ch BSS using the EM algorithm in reverberant environment, in: Proc. IEEE Workshop on Applications of Signal Processing to Audio and Acoustics (WASPAA), 2007, pp. 147-150. 
[7] C. Faller, J. Merimaa, Source localization in complex listening situations: Selection of binaural cues based on interaural coherence, Journal of the Acoustical Society of America 116 (5) (2004) 3075-3089.

[8] H. Viste, G. Evangelista, On the use of spatial cues to improve binaural source separation, in: Proc. Int. Conf. on Digital Audio Effects (DAFx), 2003, pp. 209-213.

[9] J. Mouba, S. Marchand, A source localization/separation/respatialization system based on unsupervised classification of interaural cues, in: Proc. Int. Conf. on Digital Audio Effects (DAFx), 2006, pp. 233-238.

[10] B. Loesch, B. Yang, Source number estimation and clustering for underdetermined blind source separation, Proc. 11th Int. Workshop on Acoustic Echo and Noise Control (IWAENC).

[11] H. Sawada, S. Araki, R. Mukai, S. Makino, Grouping separated frequency components by estimating propagation model parameters in frequency-domain blind source separation, IEEE Transactions on Audio, Speech, and Language Processing 15 (5) (2007) 1592-1604.

[12] M. Mandel, D. Ellis, T. Jebara, An EM algorithm for localizing multiple sound sources in reverberant environments, in: Neural Information Processing Systems, 2006, pp. 953-960.

[13] F. Nesta, P. Svaizer, M. Omologo, Cumulative state coherence transform for a robust two-channel multiple source localization, in: Proc. 8th Int. Conf. on Independent Component Analysis and Signal Separation (ICA), 2009, pp. 290-297.

[14] Z. El Chami, A. Guerin, A. Pham, C. Servière, A phase-based dual microphone method to count and locate audio sources in reverberant rooms, in: Proc. IEEE Workshop on Applications of Signal Processing to Audio and Acoustics (WASPAA), 2009, pp. 209-212.

[15] C. Liu, B. C. Wheeler, Jr, R. C. Bilger, C. R. Lansing, A. S. Feng, Localization of multiple sound sources with two microphones, Journal of the Acoustical Society of America 108 (4) (2000) 1888-1905.

[16] A. Brutti, M. Omologo, P. Svaizer, Comparison between different sound source localization techniques based on a real data collection, in: Proc. 2nd Joint Workshop on Hands-Free Speech Communication and Microphone Arrays (HSCMA), 2008, pp. 69-72. 
[17] B. Loesch, B. Yang, Comparison of different algorithms for acoustic source localization, in: ITG Fachtagung Sprachkommunikation, 2010.

[18] C. Blandin, E. Vincent, A. Ozerov, Multi-source TDOA estimation using SNR-based angular spectra, in: Proc. IEEE Int. Conf. on Acoustics, Speech, and Signal Processing (ICASSP), 2011, pp. 2616-2619.

[19] S. Arberet, R. Gribonval, F. Bimbot, A robust method to count and locate audio sources in a multichannel underdetermined mixture, IEEE Transactions on Signal Processing 58 (1) (2010) 121-133.

[20] Y. Deville, M. Puigt, Temporal and time-frequency correlation-based blind source separation methods. Part I: Determined and underdetermined linear instantaneous mixtures, Signal Processing 87 (2007) 374407.

[21] P. Bofill, Identifying single source data for mixing matrix estimation in instantaneous blind source separation, in: Proc. 18th Int. Conf. on Artificial Neural Networks (ICANN), 2008, pp. 759-767.

[22] A. Ozerov, C. Févotte, Multichannel nonnegative matrix factorization in convolutive mixtures for audio source separation, IEEE Transactions on Audio, Speech, and Language Processing 18 (3) (2010) 550-563.

[23] N. Q. K. Duong, E. Vincent, R. Gribonval, Under-determined reverberant audio source separation using a full-rank spatial covariance model, IEEE Transactions on Audio, Speech, and Language Processing 18 (7) (2010) 1830-1840.

[24] B. Loesch, B. Yang, Adaptive segmentation and separation of determined convolutive mixtures under dynamic conditions, in: Proc. 9th Int. Conf. on Latent Variable Analysis and Signal Separation (LVA/ICA), 2010, pp. 41-48.

[25] E. Vincent, S. Arberet, R. Gribonval, Underdetermined instantaneous audio source separation via local Gaussian modeling, in: Proc. 8th Int. Conf. on Independent Component Analysis and Blind Source Separation (ICA), 2009, pp. $775-782$.

[26] S. Arberet, A. Ozerov, R. Gribonval, F. Bimbot, Blind spectral-GMM estimation for underdetermined instantaneous audio source separation, in: Proc. 8th Int. Conf. on Independent Component Analysis and Blind Source Separation (ICA), 2009, pp. 751-758. 
[27] N. Roman, D. Wang, G. Brown, Speech segregation based on sound localization, Journal of the Acoustical Society of America 114 (4) (2003) $2236-2252$.

[28] H. Krim, M. Viberg, Two decades of array signal processing research: the parametric approach, IEEE Signal Processing Magazine 13 (4) (1996) 67-94.

[29] S. Araki, T. Nakatani, H. Sawada, S. Makino, Stereo source separation and source counting with MAP estimation with Dirichlet prior considering spatial aliasing problem, in: Proc. 8th Int. Conf. on Independent Component Analysis and Blind Source Separation (ICA), 2009, pp. $742-750$.

[30] A. P. Dempster, N. M. Laird, D. B. Rubin, Maximum likelihood from incomplete data via the EM algorithm, Journal of the Royal Statistical Society. Series B (Methodological) 39, No. 1 (1977) 1-38.

[31] E. Vincent, S. Araki, P. Bofill, The 2008 signal separation evaluation campaign: A community-based approach to large-scale evaluation, in: Proc. 8th Int. Conf. on Independent Component Analysis and Signal Separation (ICA), 2009, pp. 734-741.

[32] C. van Rijsbergen, Information retrieval, 2nd Edition, Butterworths, London, UK, 1979. 